Article

\title{
Polysaccharide-Based Hydrogels: The Key Role of Water in Affecting Mechanical Properties
}

\author{
Daniela Pasqui, Milena De Cagna and Rolando Barbucci * \\ Interuniversity Research Centre for Advanced Medical Systems (C.R.I.S.M.A.), Viale Matteotti 15, \\ Colle di Val d'Elsa Siena 53034, Italy; E-Mails: pasqui3@unisi.it (D.P.); \\ milena.decagna@virgilio.it (M.D.C.) \\ * Author to whom correspondence should be addressed; E-Mail: rolando.barbucci@unisi.it; \\ Tel.: +39-0577-900-327; Fax: +39-0577-900-322.
}

Received: 20 July 2012; in revised form: 27 July 2012 / Accepted: 1 August 2012 /

Published: 21 August 2012

\begin{abstract}
Different hydrogels were prepared starting from natural or semi-synthetic polysaccharides (carboxymethylcellulose, hyaluronic acid and chitosan) which were cross-linked by the addition of a cross-linking agent chosen according to the chemical groups present along the polymer chains. The cross-linking reaction allows for the formation of a three-dimensional network made of covalent bonds between the polymer chains, which is stable under physiological conditions. The presence of a substantial amount of water within the polysaccharide matrices makes such systems unique among hydrophilic gels. Water itself is responsible for some of their peculiar characteristics, one of which is their injectability which makes these hydrogels suitable for using as matrices for mini-invasive surgery and localized therapy.
\end{abstract}

Keywords: hydrogels; water molecules; injectability; rheological properties

\section{Introduction}

The use of natural polymers, or biopolymers, as materials is not new. For instance, in nature, cellulose has always been used to provide structure for plants, chitin as the exoskeleton of crawfish and shrimps, collagen for mechanical support in connective tissues, and silk in spider's webs. At present, these types of materials are the subject of much interest as materials for biomedical applications due to their high biocompatibility and low disposal costs $[1,2]$. Furthermore, the versatility of their chemical 
structures allows for the development of advanced functionalized materials that can meet a variety of requirements. In the biomedical field, the degradation of natural polymers into physiological metabolites makes them excellent candidates for a wide range of applications, such as drug delivery or regenerative medicine. Among the biopolymers, polysaccharides in particular have some excellent properties which make them the polymer group with the longest-standing and widest-ranging experience in terms of medical applications [3,4]: Non-toxicity (monomer residues are likewise not hazardous to health); water solubility or high capacity for swelling induced by simple chemical modifications and a wide variety of chemical structures [5-7]. Hyaluronic acid, carboxymethylcellose and chitosan were chosen as starting polysaccharides for the preparation of biocompatible hydrogels for biomediacal applications. Hyaluronan (Hyal) is a high molecular weight glycosaminoglycan composed of disaccharide repeating units of $\mathrm{N}$-acetylglucosamine and glucuronic acid linked together through alternating beta-1,4 and beta-1,3 glycosidic bonds. In the human body, Hyal occurs in the salt form, hyaluronate, and is found in high concentrations in several soft connective tissues, including skin, umbilical cord, synovial fluid, and vitreous humor [8-10]. Sodium carboxymethylcellulose (CMC-Na) is synthesized by swelling cellulose with sodium hydroxide and alkali-catalyzed reaction of cellulose with chloroacetic acid. Over the years, CMC has been utilized as the sole material or in combination with drugs and co-excipients in wound dressing for the treatment of partial thickness wounds, deep diabetic foot ulcers, pressure sores, surgical wounds, toxic epidermal necrolysis, and as a dermal filler [11,12]. Chitosan, a cationic group-containing polysaccharide, is obtained by means of deacetylation of chitin extracted from the external skeleton (shell) of marine crustaceans. Coming from a natural, renewable, nontoxic and biodegradable source, it is justifiably viewed as an 'ecologically-friendly' product $[13,14]$. Its quality and properties depend on its purity, degree of deacetylation (ranging between $70 \%$ and 95\%), molecular weight and crystallinity [15]. Among its applications, mention should be made of chitosan's use as a hypocholesteremic and bacteriostatic agent, as a drug carrier or as a scaffold for cell cultures [16,17].

Among hydrogels, injectable hydrogels have been attracting much interest in biomedical fields, especially for minimally-invasive surgery with the aim of avoiding the complications that may occur during traditional surgeries. There are several methods for obtaining in situ formation of a hydrogel starting from an injectable polymer solution, but all of these share the limitation of lack of control over the characteristics and stoichiometry of the hydrogels thus formed. Moreover, all the precursors added to the polymer solution remain in the injection site and may cause uncontrolled side reactions or local toxicity $[18,19]$. Our polysaccharide-based hydrogels can be considered "injectable hydrogels" thanks to their property to be squeezed through the needle of a syringe. Their injectability comes out from the fact that they show a thixotropic nature [20,21]. Hyal, CMC and CHT hydrogels, once pre-synthesized can be extensively purified in order to remove all impurities and fully characterized. They can be easily squeezed through a syringe needle, offering the advantage of opening up new perspectives in minimally-invasive surgery by using a pre-formed safe material with well known chemical and mechanical properties [7]. Localized treatment with the hydrogel improved the healing of cartilage lesions without inducing inflammatory effects [22,23].

Water is the main component of these polysaccharide-based hydrogels (more than $90 \%$ of their weight when in their swollen state). Generally, water inside hydrogel matrices can be classified as one of three types: the water in the outermost layer is called free water, and can be easily removed under 
temperate conditions as it is physically entrapped within the polymer network. Free interstitial water does not take part in hydrogen bonds with polymer molecules. Its transition temperature, enthalpy and DSC curves are similar to those of pure water. The second type is bound water, which is directly bound to the polymer chain through hydrophilic groups or via hydrogen bonds. It is an integral part of the hydrogel structure and cannot easily separated from the hydrogel. The last type is semi-bound water, with intermediate properties between those of bound and free water [24]. This paper deals with the physico-chemical characterization of the polysaccharide based hydrogels, focused to understand the role of water molecules in affecting the structure and the properties of the material in terms of swelling degree, rheological properties and infrared analysis either in the native state or after a mechanical stress.

\section{Results and Discussion}

\subsection{Hydrogel Synthesis}

Hydrogel synthesis follows the same chemical route that has been well-documented in carbodiimide chemistry [7,25]. Basically, the synthesis of CMC- and Hyal-based hydrogels exploits the formation of an amide bond between the carboxylate groups of the polymer chains and the primary amine groups of 1,3 diaminopropane (DAP), which is added to the polymer aqueous solution as the cross-linking agent of the polymer chain. 1-ethyl-3-[3-(dimethyl-amino)propyl] carbodiimide hydrochloride (EDC) works as the activating agent of the carboxylic groups, and N-hydroxysuccinimide (NHS) as the proton exchanger [26]. The reaction schemes of CMC and Hyal hydrogel are shown in Figure 1a,b. The stoichiometry of the reaction can be tuned in order to form hydrogels with different degrees of cross-linking, taking into consideration the number of carboxylic groups in each polysaccharide, i.e., one carboxylic group per disaccharide unit in Hyal and two in CMC polymer. On the other hand, CHT synthesis (see Figure 1c) involves the formation of an amide bond between the amine groups of the polymer and the carboxylic groups of the cross-linking agent, i.e., adipic acid (AA) [7]. The determination of the degree of cross-linking (defined as the number of polymer groups bound to the cross-linker) is obtained by pontentiometric titration of the free protonable groups remaining after hydrogel formation (carboxylate groups for CMC, Hyal or amine groups for CHT polymer), or by means of ${ }^{13} \mathrm{C}$ nuclear magnetic resonance [27]. Indeed, even if the molar ratio of polymer, EDC and DAP used for the synthesis of hydrogels, should allow the formation of a hydrogel cross-linked at $100 \%$, the cross-linking degree of CMC, Hyal and CHT hydrogels was $79 \%, 45 \%$ and $44 \%$ respectively as determined by potentiometric titrations. 
Figure 1. Reaction scheme of (a) Carboxy Methyl Cellulose (CMC) hydrogel;

(b) Hyaluronan (Hyal) hydrogel; and (c) Chitosan (CHT) hydrogel.

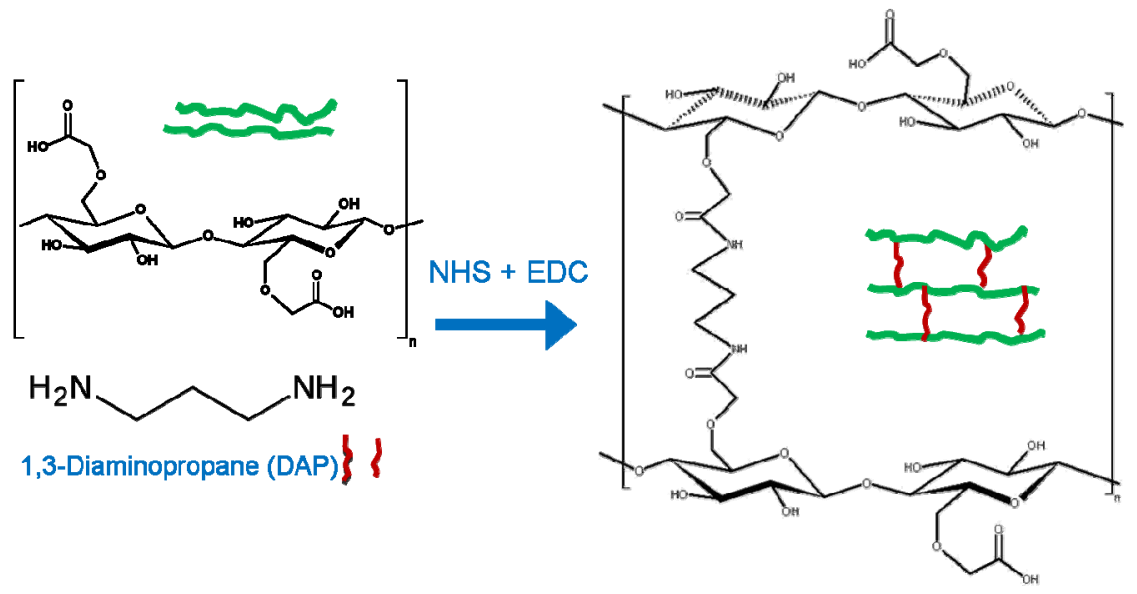

(a)

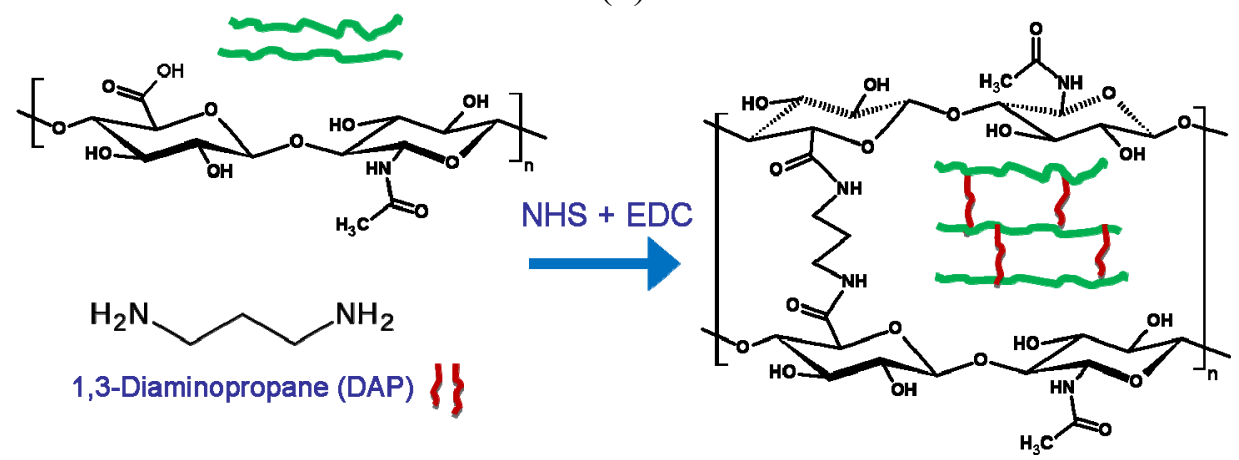

(b)

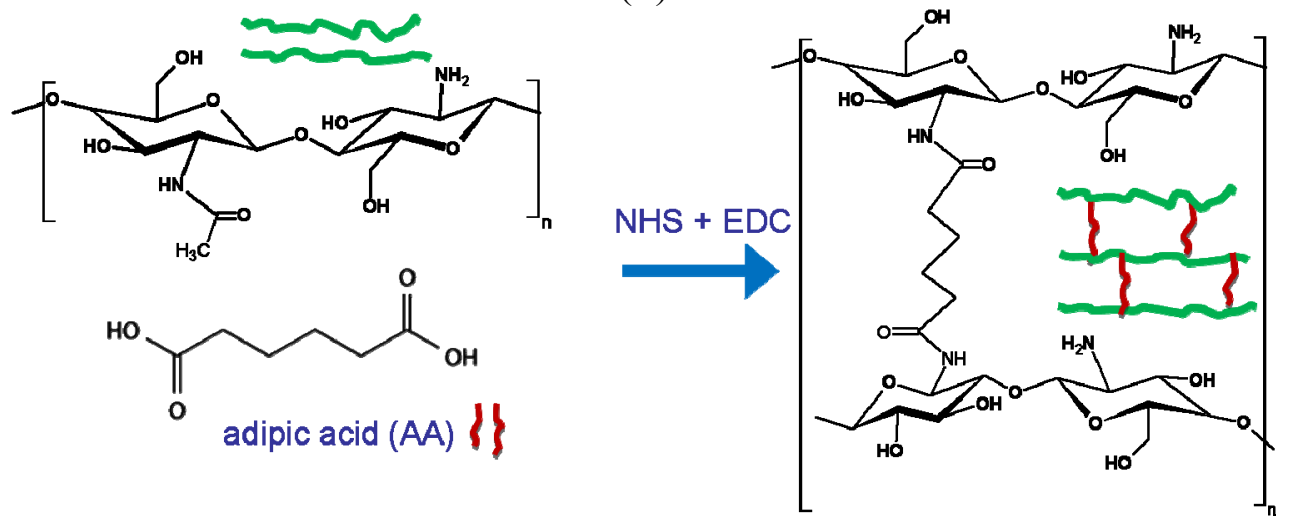

(c)

\subsection{Rheological Characterization and Swelling Degree}

In order to obtain information about the mechanical properties of the polymer network we subjected the hydrogel to a shear stress applied by a rheometer. The typical trend of G' and G' vs. oscillation stress for these polysaccharide hydrogels is plotted in Figure 2. For low values of oscillation stress, the storage modulus (G') is higher than the viscous one (G') indicating the gel-like nature of the material. Above the cross-point (i.e., the oscillation stress at which G' and G', assume the same value), the viscous modulus is higher than the storage modulus, which is characteristic of a liquid. This implies a sol-gel transition of the material [20]. The behavior of G' and G" was studied by increasing and 
decreasing the applied stress cyclically on the same hydrogel sample. When the oscillation stress is decreased (step 2 Figure 2), G' and G'" show always lower values than those found during the increase in oscillation stress (step 1 Figure 2). At the end of step 2, the G' and G" values are lower than those of the native hydrogel at the first step. By repeating the same sequence of steps several times (that is, increasing and decreasing the oscillation stress on the same sample, step 3 and step 4 Figure 2), G' and G" values follow the same trend and maintain the same values as those determined in step 2. This result indicates a sort of "recovery" effect of the G' and G" values once the material is subjected to further cycles of stresses [7,26]. In the case of squeezed hydrogels (Figure 3(a-c)), both the storage modulus G' and the loss modulus G' values are reduced due to the passage from the syringe with respect to those of the native hydrogels (Table 1). However, by repeating the same cycle of stress several times on the squeezed hydrogels, as done for the native ones (see Figure 2), G' and G" values remain the same. This does not occur for a native hydrogel (see step 1 and step 2 Figure 2). The squeezing process induces the same effect on the hydrogel as the stress applied by the rheometer. The lower values of G' and G' of a squeezed hydrogel with respect to its native sample indicate that the hydrogel network is softened by the application of a shear stress such as squeezing through a syringe or by the stress applied by a rheometer. However, the application of an additional stress does not lead to further alterations of the hydrogel's mechanical properties. Hence, the squeezed hydrogels behave in the same manner as native hydrogels after the application of a stress applied by a rheometer.

Figure 2. Typical trend of $G^{\prime}$ and G" values vs. oscillation stress during sweep test performed on a polysaccharide-based hydrogel. The figure shows the G' and G" value vs. oscillation stress for each step of the test.

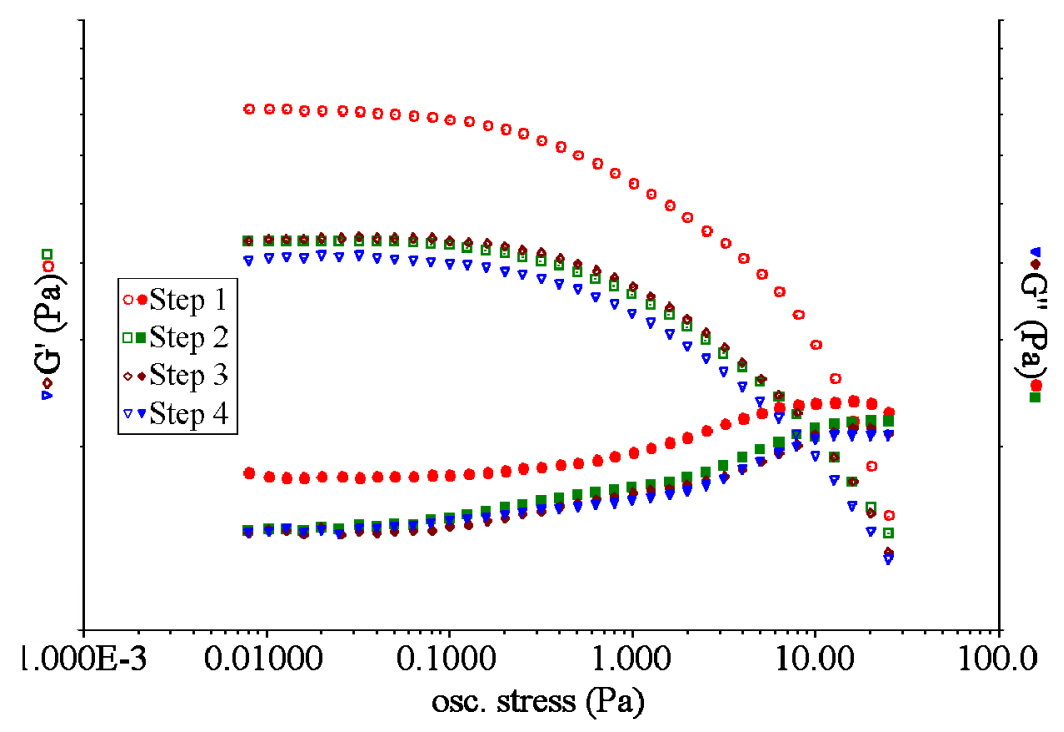


Figure 3. Stress sweep test performed on (a) CMC hydrogel; and (b) Hyal hydrogel; (c) CHT hydrogels, native and after being squeezed through a syringe. The figure shows the G' and G" values vs. oscillation stress for each step of the test.

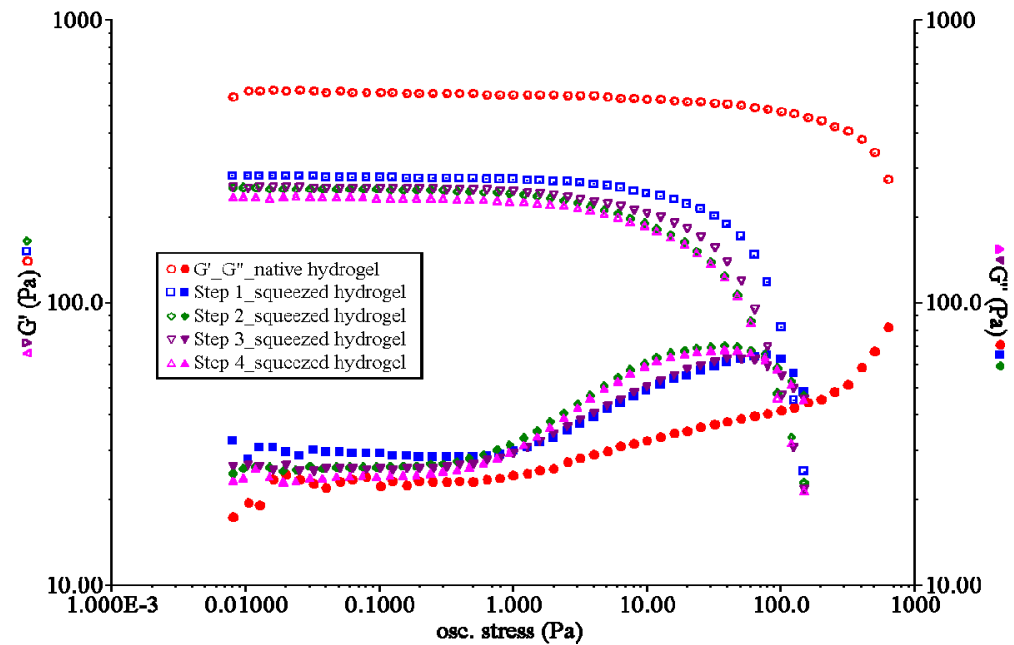

(a)

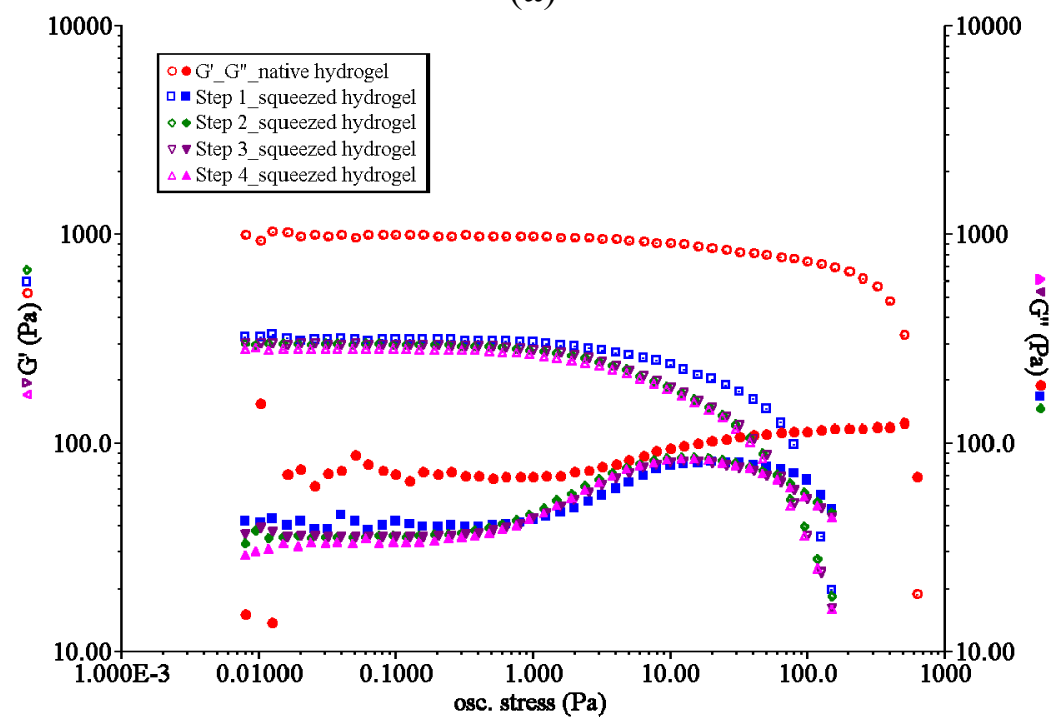

(b)

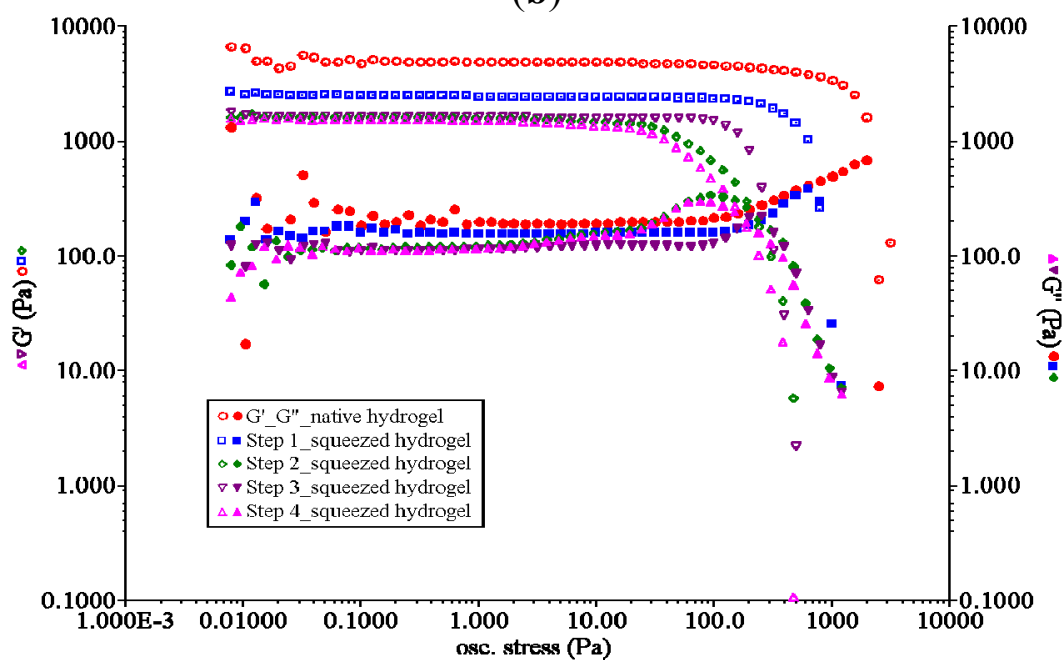

(c) 
Table 1. Storage modulus (G') and elastic modulus (G”) of polysaccharide hydrogels in native and after being squeezed. Data are reported as mean of three values \pm standard error.

\begin{tabular}{cccc}
\hline Hydrogel type & Status & $\mathbf{G}^{\prime}(\mathbf{P a})$ & $\mathbf{G}^{\prime}(\mathbf{P a})$ \\
\hline \multirow{2}{*}{ CMC } & Native & $550 \pm 30$ & $25 \pm 1$ \\
& Squeezed & $240 \pm 20$ & $20 \pm 2$ \\
\multirow{2}{*}{ HYAL } & Native & $970 \pm 25$ & $65 \pm 10$ \\
& Squeezed & $340 \pm 20$ & $45 \pm 5$ \\
\multirow{2}{*}{ CHT } & Native & $4350 \pm 650$ & $165 \pm 45$ \\
& Squeezed & $3460 \pm 150$ & $230 \pm 15$ \\
\hline
\end{tabular}

Infrared spectra and nuclear magnetic resonance (N.M.R.) of the native hydrogel and that of the same hydrogel once stressed appear to be overlapped, indicating that the hydrogel's chemistry is maintained. Therefore passing through a syringe or being subjected to a stress applied by a rheometer does not alter the chemical structure or affect the degree of cross-linking of the hydrogel [26]. We can say that the application of a stress induces a change in the hydrogel's matrix without affecting its chemistry. Therefore, the involvement of different factors such as the amount of water contained within the hydrogel and its arrangement as well as the polymer chains disposal have been considered.

The amount of water contained in the hydrogels was determined by measurements of swelling in $\mathrm{NaCl} 0.1 \mathrm{M}$ solution at $\mathrm{pH} 7.4$ (see Table 2). All the native hydrogels uptake the same amount of water at their maximum swelling degree within the experimental errors even if the structure of the starting polymers and the cross-linking of the hydrogels are different from each other. Concerning the swelling degree of squeezed hydrogels, the value of Hyal is slightly higher than that of CMC. CHT shows the lowest swelling degree (see Figure 4). This trend can be explained by considering the pKa of polysaccharides. The CHT hydrogel, which contains free amine groups, is partially charged at $\mathrm{pH}$ 7.4, as the pKa of CHT is between 6.5 and 7.5, depending on the degree of deacetylation [28]. $\mathrm{The}^{\mathrm{NH}_{3}}{ }^{+}$ groups, which are highly hydrophilic, interact with the remaining free $\mathrm{NH}_{2}$, collapsing the polymeric chains and hindering the solvation of the hydrogel. In fact, the swelling degree of CHT is significantly higher $(1000)$ in $\mathrm{NaCl} 0.1 \mathrm{M}$ at $\mathrm{pH} 4$, when all the free $-\mathrm{NH}_{2}$ groups are protonated and able to repel each other. The $\mathrm{CMC}$ and Hyal hydrogels are both negatively charged at $\mathrm{pH} 7.4$ because of the presence of free, completely unprotonated $\mathrm{COO}^{-}$(the pKa of both Hyal and CMC is 4.0-4.5) [29]. The presence of negatively charged groups increases the electrostatic repulsions between the polymer chains and thus the amount of water that can enter the hydrogel.

Table 2. Swelling degree of native and squeezed hydrogels in $\mathrm{NaCl} 0.1 \mathrm{M}$ solution. The reported data represent the average of three samples \pm standard error.

\begin{tabular}{ccc}
\hline Hydrogel type & Status & Swelling degree \% \\
\hline \multirow{2}{*}{ CMC } & Native & $640 \pm 50$ \\
& Squeezed & $1235 \pm 125$ \\
HYAL & Native & $720 \pm 25$ \\
& Squeezed & $1300 \pm 150$ \\
CHT & Native & $650 \pm 80$ \\
& Squeezed & $830 \pm 125$ \\
\hline
\end{tabular}


Figure 4. Swelling degree of different squeezed polysaccharide-based hydrogels in $0.1 \mathrm{M}$ $\mathrm{NaCl}$ solution at $\mathrm{pH}$ 7.4. Data are reported as mean of three values \pm standard error.

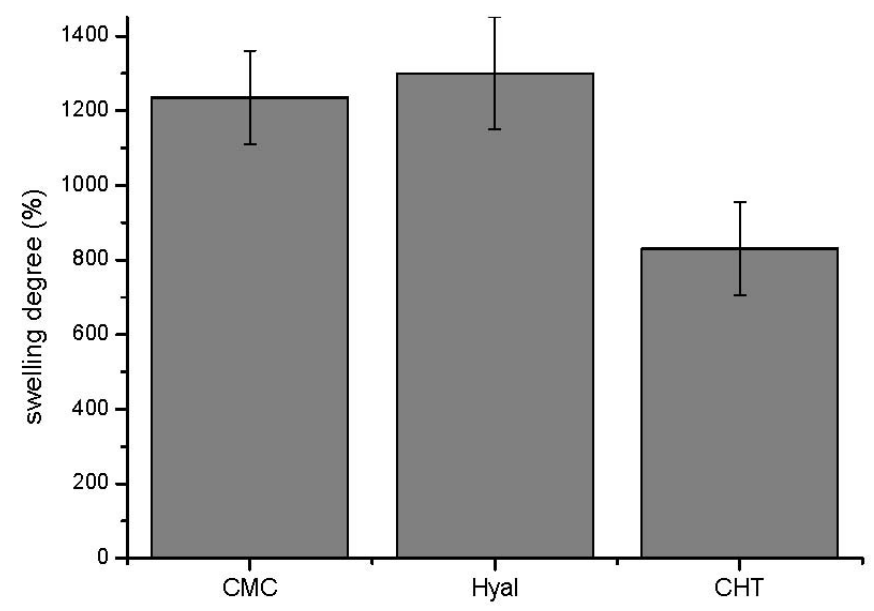

The total amount of water within hydrogels in $\mathrm{NaCl} 0.1 \mathrm{M}$ is higher for the squeezed hydrogels than for the native ones (see Table 2). Under the application of a shear stress the polymer chains which are entangled during the cross-linking reaction unroll and align along the direction of the stress [30,31]. Hence, this necessarily induces a new disposal of water molecules within the hydrogel network. Bound water molecules move to a semi-bound state (see FT-IR analyses). This movement of water molecules determines a decrease of the mechanical properties of the hydrogel. The change in the arrangement of water molecules seems to be irreversible. In fact, by dipping a squeezed hydrogel in water for several days, it does not regain the original mechanical properties before being squeezed. The passage of water molecules from bound state to a semi bound state make the material also capable to uptake a higher amount of water because the higher mobility of water molecules allow them to form a higher number of bonds with other free water molecules.

\subsection{FT-IR Analysis: The Role of Water}

The role of water and water interactions inside the hydrogel were investigated by means of FT-IR analyses of the hydrogel in both swollen and dried forms. A typical IR spectrum of CMC hydrogel in dried state is shown in Figure 5, with main peak assignment listed in Table 3. In a first approximation, the spectrum is composed of four regions $[32,33]$ :

1. An intense band in the region between 950 and $1200 \mathrm{~cm}^{-1}$ corresponding to the $-\mathrm{C}-\mathrm{O}, \mathrm{C}-\mathrm{CH}$ stretching and $\mathrm{CO}$ and $\mathrm{C}-\mathrm{O}-\mathrm{C}$ bending of the glycoside ring.

2. A region around 1300 and $1450 \mathrm{~cm}^{-1}$ where bands related to $\mathrm{C}-\mathrm{CH}$ and $\mathrm{O}-\mathrm{CH}$ stretching and -CO stretching are observed together with a peak at $1408 \mathrm{~cm}^{-1}$ beloning to the symmetric stretching of the free carboxylate groups of the polymer not involved in the hydrogel formations.

3. An intense group of bands that extends from 1500 to $1700 \mathrm{~cm}^{-1}$ corresponding to the amide I, amide II and free carboxylate groups of the CMC polymer.

4. A broad and intense band in the region between 2500 and $3600 \mathrm{~cm}^{-1}$ which is composed of several bands ascribed to the $-\mathrm{OH}$ stretching of the polymer glycoside ring, and the $-\mathrm{OH}$ stretching of water free and involved in hydrogel bonds. 
Figure 5. Infrared spectrum of native CMC hydrogel in the region between 4000 and $750 \mathrm{~cm}^{-1}$.

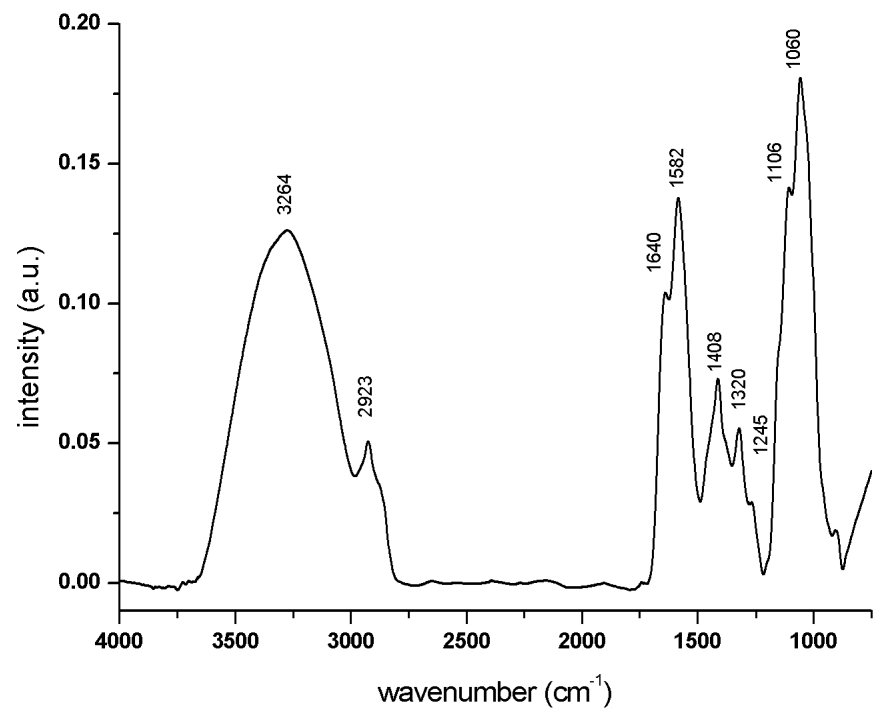

Table 3. Peak wavenumbers $\left(\mathrm{cm}^{-1}\right)$ and assignments for CMC hydrogel.

\begin{tabular}{cc}
\hline Wavenumber $\left(\mathbf{c m}^{-\mathbf{1}}\right)$ & Assignment \\
\hline 3264 & water molecules $-\mathrm{OH}$ \\
2923 & $-\mathrm{C}-\mathrm{C}-\mathrm{C}-\mathrm{H}$ stretch \\
1640 & amide $-\mathrm{C}=\mathrm{O}$ stretch \\
1582 & carboxylate symm. stretch \\
1408 & carboxylate asymm. stretch \\
1320 & C-CH and O-CH stretch \\
1245 & $-\mathrm{CO}$ stretch \\
1106 & $\mathrm{C}-\mathrm{C} \mathrm{C}-\mathrm{O}$ stretch \\
1060 & $\mathrm{C}-\mathrm{O}-\mathrm{C}$ bend \\
\hline
\end{tabular}

Our attention was focused on the spectral region between 1500 and $1800 \mathrm{~cm}^{-1}$. In order to determine the components contributing to the band envelopes, we performed a curve-fitting analysis of the spectra of the swollen hydrogel in the region between 1500 and $1800 \mathrm{~cm}^{-1}$ (see Figure 6). The assignment of peak components for the CMC hydrogel in its native state and after squeezing is reported in Table 4. The main difference regards the position of the peak ascribed to the carboxylate asymmetric stretching, which shifts from $1578 \mathrm{~cm}^{-1}$ (spectrum of native hydrogel) to $1590 \mathrm{~cm}^{-1}$ (spectrum of squeezed hydrogel). The shift to higher wavenumbers is attributed to the loss of some hydrogen bonds involving the free carboxylate groups of the polymer chains [34]. No significant shifts were observed in the position of amide I in the spectrum of native and squeezed CMC hydrogel in their swollen state. However, once the hydrogel is left to dry, the amide I peak shifts to higher wavenumbers compared with the position of the same peak for the hydrogel in the swollen state (from $\sim 1631 \mathrm{~cm}^{-1}$ to $\sim 1645 \mathrm{~cm}^{-1}$ ). This indicates that in the dried state, additional interactions between amide groups are involved [34,35]. 
Figure 6. Curve-fitting analysis of the spectrum of swollen CMC hydrogel in native state. The components contributing to the peak were fitted using a mixture of Lorentzian and Gaussian line shapes. The component frequency, the full width, half, maximum and peak areas were optimized in the fitting procedure. The thick line represents the overall fit to four components.

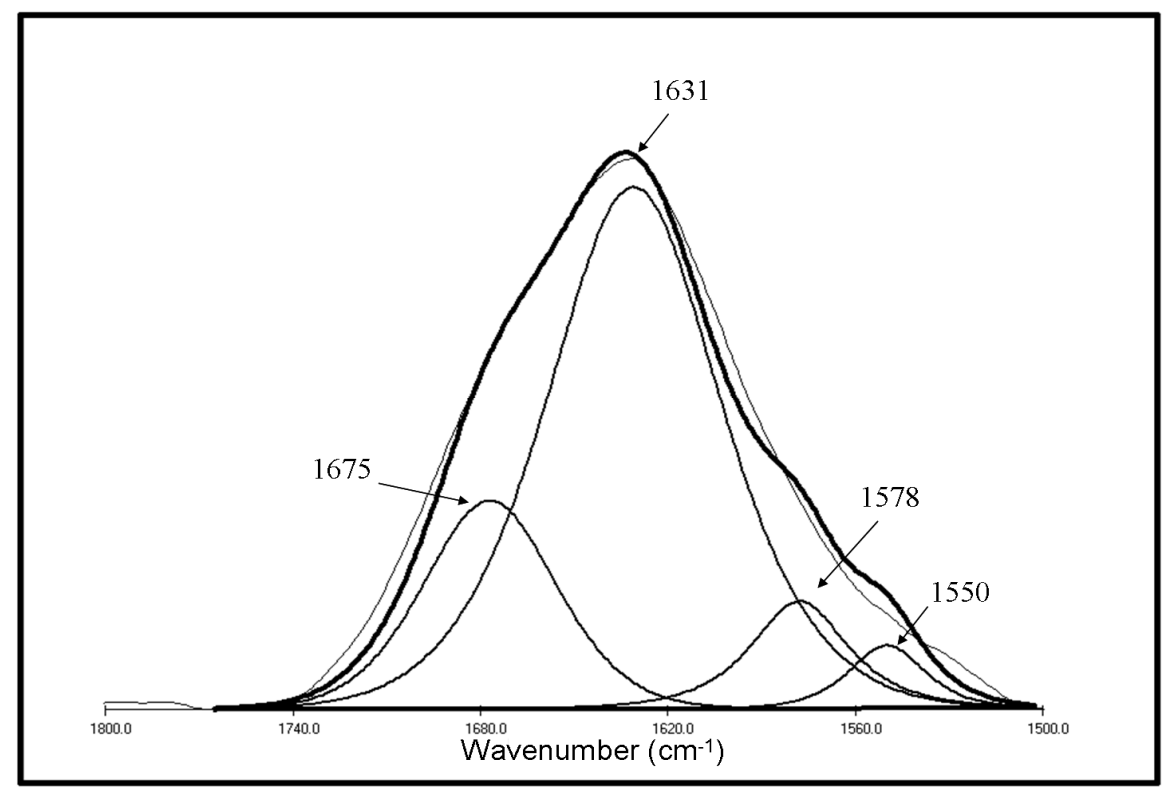

Table 4. Assignment of bands of spectra for CMC hydrogels (a) native and (b) squeezed in swollen state present in the region between 1700 and $1500 \mathrm{~cm}^{-1}$.

\begin{tabular}{cccc}
\hline $\begin{array}{c}\text { CMC hydrogel } \\
\text { swollen state }\end{array}$ & $\begin{array}{c}\text { Main peak wavenumber } \\
\left(\mathbf{c m}^{-\mathbf{1}}\right)\end{array}$ & Wavenumber $\mathbf{( \mathbf { c m } ^ { - \mathbf { 1 } } )}$ & Assignment \\
\hline \multirow{3}{*}{ native } & & 1675 & $\begin{array}{c}\text { water molecules }-\mathrm{OH} \text { bend } \\
\text { amide }-\mathrm{C}=\mathrm{O} \text { stretch. }\end{array}$ \\
& 1631 & 1631 & carboxylate asymmetry stretch. \\
\cline { 2 - 4 } & 1550 & 1578 & amide $-\mathrm{N}-\mathrm{H}$ bend \\
\hline \multirow{3}{*}{ squeezed } & 1632 & 1675 & water molecules $-\mathrm{OH}$ bend \\
& & 1631 & amide $-\mathrm{C}=\mathrm{O}$ stretch. \\
& 1552 & 1590 & carboxylate asymm. stretch. \\
\cline { 2 - 4 } & & 1552 & amide $-\mathrm{N}-\mathrm{H}$ bend \\
\hline
\end{tabular}

The same behavior was found for Hyal hydrogels. The typical IR spectrum of Hyal hydrogel in dried state is reported in Figure 7, with the main peak assignments listed in Table 5. As made for CMC hydrogel, a curve-fitting analysis of the swollen hydrogel spectra in the region between 1500 and $1800 \mathrm{~cm}^{-1}$ was carried out. The asymmetric stretching of free carboxylate groups shifted from $1608 \mathrm{~cm}^{-1}$ in the spectrum of native swollen hydrogel to $1616 \mathrm{~cm}^{-1}$ in the spectrum of squeezed hydrogel. The shift of the carboxylate groups is again attributed to the presence of hydrogen bonds involving the carboxylate groups. The higher amount of hydrogen bonds [36] in the native hydrogels could be responsible for the higher storage modulus ( $\left.G^{\prime}\right)$ and viscous modulus (G') values of the native $\mathrm{CMC}$ and Hyal hydrogels in comparison with the same squeezed hydrogels. 
Figure 7. Infrared spectrum of native Hyal hydrogel in the region between 4000 and $750 \mathrm{~cm}^{-1}$.

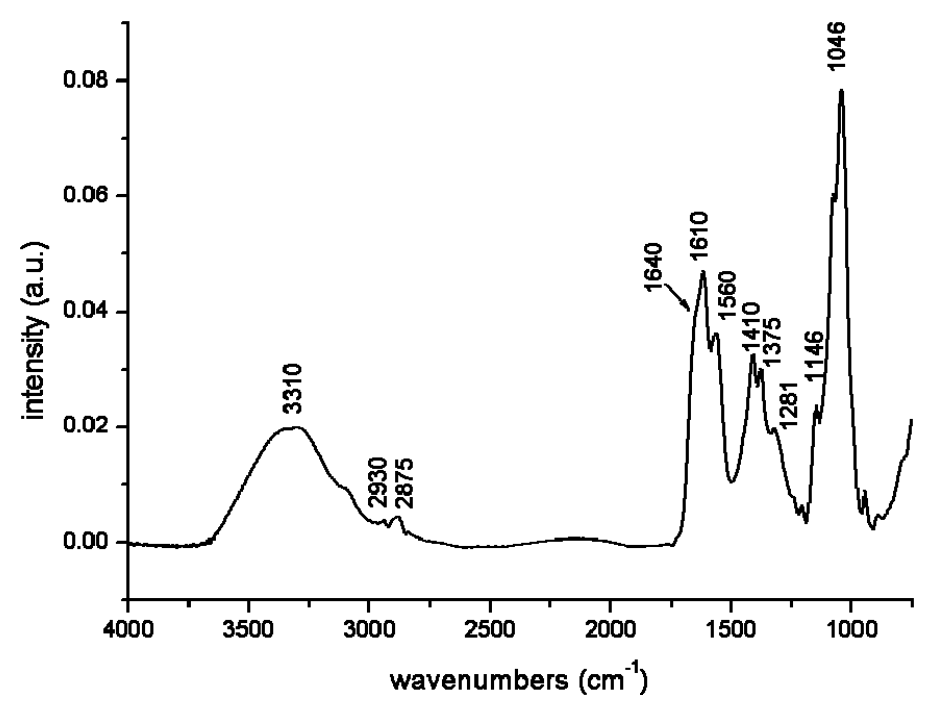

Table 5. Peak wavenumbers $\left(\mathrm{cm}^{-1}\right)$ and assignments for Hyal hydrogel.

\begin{tabular}{cc}
\hline Wavenumber $\left(\mathbf{c m}^{-\mathbf{1}}\right)$ & Assignment \\
\hline 3310 & water molecules $-\mathrm{OH}$ \\
2930 & $-\mathrm{C}-\mathrm{C}-\mathrm{C}-\mathrm{H}$ stretch \\
2875 & $-\mathrm{C}-\mathrm{C}-\mathrm{C}-\mathrm{H}$ stretch \\
1640 & amide $-\mathrm{C}=\mathrm{O}$ stretch \\
1610 & carboxylate asymm. stretch \\
1560 & amide $\mathrm{N}-\mathrm{H}$ bend \\
1410 & carboxylate symm. stretch \\
1375 & C-CH and O-CH stretch \\
1281 & $-\mathrm{C}-\mathrm{O}$ stretch \\
1146 & $\mathrm{C}-\mathrm{C}$ C-O stretch \\
1046 & $\mathrm{C}-\mathrm{O}-\mathrm{C}$ bend \\
\hline
\end{tabular}

The spectrum of CHT hydrogel is shown in Figure 8 and the assignment of the main peaks is shown in Table 6. As discussed above for $\mathrm{CMC}$ hydrogel, the entire spectrum can be divided into four regions: An intense band in the region between 950 and $1200 \mathrm{~cm}^{-1}$ corresponding to $-\mathrm{C}-\mathrm{O}, \mathrm{C}-\mathrm{CH}$ stretching and $\mathrm{CO}$ and $\mathrm{C}-\mathrm{O}-\mathrm{C}$ bending of the glycosidic ring; a region around 1300 and $1450 \mathrm{~cm}^{-1}$ where typical chitosan polymer bands related to $\mathrm{C}-\mathrm{CH}$ and $\mathrm{O}-\mathrm{CH}$ stretching and $-\mathrm{CO}$ stretching are found; and an intense group of bands that extends from 1500 to $1700 \mathrm{~cm}^{-1}$ corresponding to the amide I and amide II peaks ascribable to the two amide groups of CHT hydrogel, one belonging to chitin polymer and the other related to the one formed during the cross-linking reaction. The last region, which is composed of several bands ascribed to the $-\mathrm{OH}$ stretching of the polymer glycosidic ring, $-\mathrm{OH}$ stretching of water molecules (both free and involved in hydrogen bonds) and different types of amine group stretching, is between 2500 and $3600 \mathrm{~cm}^{-1}$ [37,38]. The spectrum of the swollen squeezed hydrogel was compared to that of the same hydrogel before squeezing in the region between 2600 and $3600 \mathrm{~cm}^{-1}$ (see Table 7) after application of the same curve-fitting procedure used for CMC and Hyal-based hydrogels. A shift of $12 \mathrm{~cm}^{-1}$ to lower wavenumbers was noted for the peaks at $3510 \mathrm{~cm}^{-1}$ and $3312 \mathrm{~cm}^{-1}$ ascribable to the stretching of free $\mathrm{NH}_{2}$ groups and the stretching of free $\mathrm{NH}_{3}{ }^{+}$groups 
respectively, in the spectrum of the squeezed swollen CHT hydrogel with respect to that of native CHT hydrogel. In the case of the peak related to the amide $\mathrm{N}-\mathrm{H}$ stretching, a shift of $8 \mathrm{~cm}^{-1}$ in position was found $\left(3130 \mathrm{~cm}^{-1}\right.$ for native hydrogels and $3138 \mathrm{~cm}^{-1}$ for squeezed hydrogels). Generally, the shift of the stretching of primary amine groups to lower wavenumbers is due to an interaction among such groups leading to the formation of $\mathrm{NH}_{2}-\mathrm{NH}_{3}{ }^{+}$clusters [39]. In the case of CHT hydrogels, such interactions seem to be stronger for the squeezed sample than for the native one (see Table 1). On the contrary, no significant difference of the positions of the amide I or amide II bands were observed in the spectrum of native and squeezed hydrogels in the swollen or dried state. Furthermore, in the case of the dried hydrogel, the peaks of $\mathrm{NH}_{2}$ groups, the stretching of free $\mathrm{NH}_{3}{ }^{+}$groups and the stretching of $\mathrm{NH}$ groups are located at 3440,3260 and $3100 \mathrm{~cm}^{-1}$ respectively, all at wavenumbers lower than those of the swollen hydrogel. This effect is again due to the existence of $\mathrm{NH}_{2}-\mathrm{NH}_{3}{ }^{+}$interactions, which are more intense when the hydrogel is dried. In contrast, when the hydrogel is in the swollen state, the $\mathrm{NH}_{2}-\mathrm{NH}_{3}{ }^{+}$interaction can be mediated by water molecules via hydrogen bonds between water and primary amine groups. A sort of competition between $\mathrm{NH}_{2}$-water, $\mathrm{NH}_{3}{ }^{+}$-water and $\mathrm{NH}_{2}-\mathrm{NH}_{3}{ }^{+}$occurs, and it seems that the interactions between amine groups and water molecules are greater in the native swollen state than in the squeezed and dried state.

Figure 8. Infrared spectrum of native CHT hydrogel in the region between 4000 and $750 \mathrm{~cm}^{-1}$.

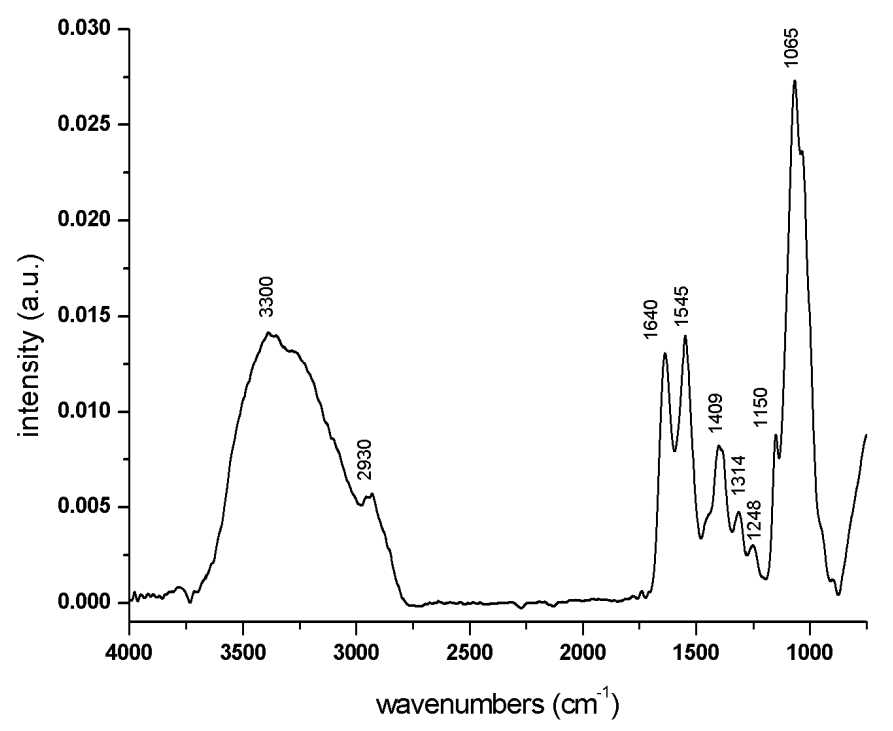

Table 6. Peak wavenumbers $\left(\mathrm{cm}^{-1}\right)$ and assignments for CHT hydrogel.

\begin{tabular}{cc}
\hline Wavenumber $\left(\mathbf{c m}^{-\mathbf{1}}\right)$ & Assignment \\
\hline 3300 & water molecules $-\mathrm{OH}$ \\
2930 & $-\mathrm{C}-\mathrm{C}-\mathrm{C}-\mathrm{H}$ stretch \\
1640 & amide $-\mathrm{C}=\mathrm{O}$ stretch. \\
1545 & amide $\mathrm{N}-\mathrm{H}$ stretch \\
1409 & $\mathrm{CH}_{2}$ bend \\
1314 & $\mathrm{C}-\mathrm{CH}$ and $\mathrm{O}-\mathrm{CH}$ stretch. \\
1248 & $-\mathrm{CO}$ stretch \\
1150 & $\mathrm{C}-\mathrm{CH} \mathrm{C}-\mathrm{O}$ stretch \\
1066 & $\mathrm{C}-\mathrm{O}-\mathrm{C}$ bend \\
\hline
\end{tabular}


Table 7. Assignment of bands of spectra for CHT hydrogels (a) native; and (b) squeezed, both in swollen state, present in the region between 3000 and $3650 \mathrm{~cm}^{-1}$

\begin{tabular}{cccc}
\hline $\begin{array}{c}\text { CHT hydrogel } \\
\text { swollen state }\end{array}$ & $\begin{array}{c}\text { Main peak wavenumber } \\
(\mathbf{c m})\end{array}$ & Wavenumber $\left(\mathbf{c m}^{-\mathbf{1}}\right)$ & Assignment \\
\hline \multirow{3}{*}{ native } & 3317 & 3620 & $-\mathrm{OH}$ bend \\
& 3130 & 3510 & $\begin{array}{l}\text { free }-\mathrm{NH}_{2} \text { stretch } \\
\text { free }-\mathrm{NH}_{3}{ }^{+} \text {stretch }\end{array}$ \\
\cline { 2 - 4 } & 3312 & amide $-\mathrm{N}-\mathrm{H}$ stretch \\
\hline \multirow{3}{*}{ squeezed } & 3300 & 3130 & $-\mathrm{OH}$ bend \\
& 3138 & 3495 & Free $-\mathrm{NH}_{2}$ stretch \\
& & 3300 & free $-\mathrm{NH}_{3}{ }^{+}$stretch \\
\cline { 2 - 4 } & 3138 & amide $-\mathrm{N}-\mathrm{H}$ stretch \\
\hline
\end{tabular}

Upon application of a shear stress, such as the passage from a syringe needle, the strength of such physical interactions decreases, making the hydrogel softer. Basically, a different arrangement of water molecules from a bound state to a semi-bound state occurs, leading to a decrease in the mechanical properties of the hydrogels (see Figure 9). In summary, the presence of water molecules organized near the polymeric chains-so-called bound and semi-bound water-influences the mechanical properties of the hydrogels, making the materials tougher or softer. In the case of native-based hydrogel, polymer-water interactions occurring via hydrogen bonds which are quite strong are responsible for the higher values of G' and G" as compared with the same squeezed hydrogel.

Figure 9. Schematic drawing showing the arrangement of water molecules inside a polysaccharide-based hydrogel in the native state and under stress.

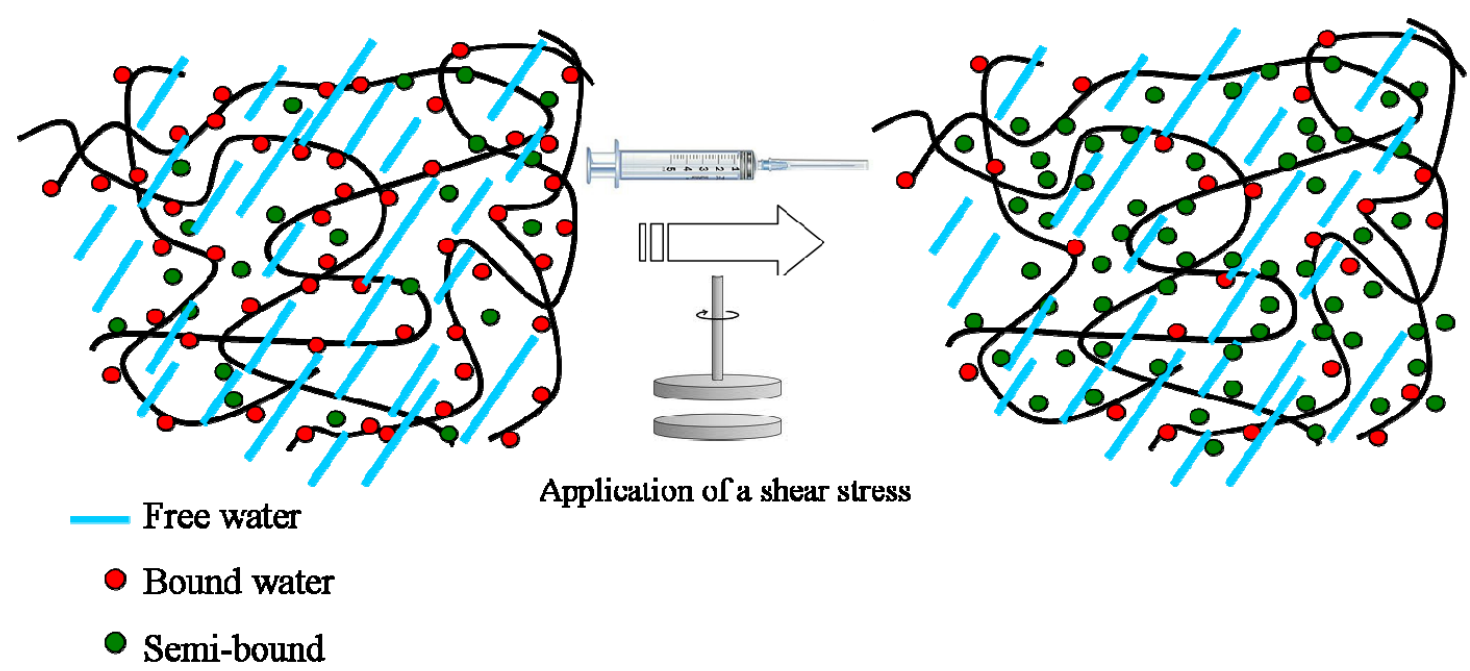

\section{Experimental Section}

\subsection{Materials}

Carboxy Methyl Cellulose with a degree of carboxymethylation of 95\% (CMC) (MW $700 \mathrm{KDa}$ ) Chitosan (MW $210 \mathrm{KDa}$ ), with a degree of deacetylation of $75 \%$ (CHT) and Hyaluronan (Hyal) (MW $200 \mathrm{KDa}$ ) were purchased from Sigma-Aldrich (Switzerland). Hyaluronic acid in the sodium salt 
form (Hyal) (MW 150-200 KDa) was purchased from Biophyl S.p.A. (Milan, Italy). The cross-linking agents, i.e., 1-3-diaminopropane (DAP) and adipic acid (AA), the activating agents 1-ethyl-3-[3-(dimethyl-amino)propyl)carbodiimide hydrochloride (EDC), N-hydroxysuccinimide (NHS) and all other chemicals and solvents were purchased from Sigma-Aldrich and used with no further purification.

\subsection{Hydrogel Synthesis}

All the hydrogels were synthesized by cross-linking each polysaccharide using an appropriate cross-linking agent chosen according to the chemical moieties present along the polysaccharide chains. The Hyal and CMC hydrogels were synthesized following the same chemical route, i.e., by exploiting the EDC chemistry: basically, an amide bond between the carboxylic groups of the polysaccharides and the primary amine of 1,3-diaminopropane (DAP) - the cross-linking agent-was formed thanks to the presence of EDC. The cross-linking agent, DAP was added to the mixture at a molar ratio of 0.5 with respect to the moles of carboxylic acid of the polymers and to EDC and NHS moles. The molar ratio of EDC and NHS mole is 1 to 1 [7]. The $\mathrm{pH}$ of the mixture was adjusted to 4.75 and the reaction was allowed to continue under stirring overnight at room temperature. The synthesis of CHT followed the same route with the use of adipic acid (AA) instead of DAP as the cross-linking agent by using the same molar ratio of EDC, NHS and cross-linker of the synthesis of Hyal and CMC hydrogels. These molar ratios have been used for the synthesis of hydrogels with a theoretical cross-linking of $100 \%$. Once formed, the hydrogel was purified by means of alternate washings in absolute ethanol and water for 4-5 days until no traces of free EDC or NHS were revealed by UV spectrophotometry.

\subsection{Mechanical Properties}

Rheological measurements were performed on native hydrogels at their maximum swollen state and the same hydrogels once being squeezed from a syringe with a needle of 18 gauge. A strain-controlled AR2000 Rheometer (TA-Instruments, Leatherhead, United Kingdom) in the parallel plate configuration at a controlled temperature of $25 \pm 1{ }^{\circ} \mathrm{C}$ was used. The native hydrogels were directly squeezed between the parallel plates of the rheometer. During a stress sweep test, the sample is subjected to an increasing or decreasing stress, while frequency and temperature are maintained constant. A frequency of $1 \mathrm{~Hz}$ was used. The test gives the values of the storage modulus $\left(\mathrm{G}^{\prime}\right)$ and the loss modulus (G”). The G' values provide information about the energy stored in the material during deformation stress while G" describes its viscous character. In order to verify how the hydrogels respond to the variation of an applied stress, a four-step dynamic stress sweep test was performed. The measurement is carried out by increasing (step 1) and decreasing (step 2) the oscillation twice, through the addition of a further increasing step (step 3) and decreasing step (step 4) [7,25]. The sample is subjected cyclically to the same applied stress (increasing and decreasing steps). Moreover, rheological measurements were performed on hydrogels once squeezed by waiting for different time from the squeezing. No variation of G' and G' values by waiting for longer time form the squeezing were observed (by keeping the sample once squeezed in a moist chamber). 


\subsection{Water Uptake}

Swelling measurements were performed on both native and squeezed hydrogels (at least three samples for each type of hydrogel) by estimating the variation in the height of each hydrogel from the dried state (freeze dried hydrogel containing about $10 \%$ of water) to the swollen state. The native and squeezed hydrogels were freeze-dried and then a same amount of dried hydrogel placed inside small cylindrical containers (diameter $12 \mathrm{~mm}$, height $35 \mathrm{~mm}$ ) and pressed down to the bottom. The initial height of each sample was $1.0 \mathrm{~mm}$. Each hydrogel was immersed in $1 \mathrm{~mL}$ of $\mathrm{NaCl} 0.1 \mathrm{M}$ solution at $\mathrm{pH} 7.4$ and the height of the swollen material was measured using a digital caliber (experimental error \pm 0.02 ) until the value remained unchanged.

The swelling degree (SD) of each hydrogel was calculated according to the equation:

$$
\left.\% \text { S. D. }=\left(\mathrm{h}_{\mathrm{s}}-\mathrm{h}_{0}\right) / \mathrm{h}_{0}\right) \times 100
$$

where $h_{s}$ is the height of the swollen hydrogel and $h_{0}$ is the initial height of the hydrogel in dried state (freeze-dried) [7].

\subsection{FT-IR Analysis}

Infrared analyses were performed on native hydrogels and on the same hydrogels immediately after being squeezed. Infrared spectra were recorded using an FTIR spectrometer (Biorad FTS 6000) working in attenuated total reflection mode equipped with a $45^{\circ}$ Ge ATR crystal and a mercury cadmium telluride detector. The spectra were an average of 512 scans at a resolution of $2 \mathrm{~cm}^{-1}$. The spectra were corrected for the baseline and smoothed. The spectrum of water was subtracted for each reordered spectrum of hydrogels in both hydrated and dried state using the same parameters. In order to find all the components of each peak, curve fitting was performed using special software (XPSPEAK). Spectra were fitted by using a mixture of Lorentzian $(20 \%)$ and Gaussian (80\%) line shapes by minimization the error sum. The spectra fitted well with four components of the main peak.

\section{Conclusions}

Polysaccharide-based hydrogels starting from polymers of varying chemistry were synthesized. All hydrogels contain huge amounts of water, which affects their rheological properties. Their most advantageous properties have to do with their injectability, which makes them promising biomaterials for minimally-invasive surgery and localized therapy. The arrangement of water molecules inside hydrogel structures can be considered a critical point in understanding their rheological behavior. From data reported in this paper, we deduce that under the application of a shear stress (such as passage through a syringe needle or the stress applied by a rheometer), a different arrangement of water molecules from a bound state to a semi-bound state occurs, leading to a decrease in the mechanical properties of the hydrogels and an increase of the swelling degree.

\section{References}

1. Kaplan, D.L. Introduction to polymers from renewable resources. In Biopolymers from Renewable Resources; Kaplan, D.L., Ed.; Springer Verlag: Berlin, Germany, 1998; pp. 1-29. 
2. Yannas, I.V. Natural materials. In Biomaterials Science. An Introduction to Materials in Medicine; Ratner, B.D., Hoffman, A.S., Schoen, F.J., Lemons, J.E., Eds.; Academic Press California: San Diego, CA, USA, 1996; pp. 84-94.

3. Coviello, T.; Matricardi, P.; Marianecci, C.; Alhaiqu, F. Polysaccharide hydrogels for modified release formulations. J. Control. Release 2007, 119, 5-24.

4. Stalling, S.S.; Akintoye, S.O.; Nicol, S.B. Development of photocrosslinked methylcellulose for soft tissue reconstruction. Acta Biomater. 2009, 5, 1911-1918.

5. Jin, R.; Moreira-Teixeira, L.S.; Krouwels, A.; Dijstra, P.J.; van Blitterswilk, C.A.; Karperien, M.; Feijen, J. Syntheisis and characterization of hyaluronic acid-poly(ethylene glycol) hydrogels via Micheal addition: An injectable biomaterial for cartilage repair. Acta Biomater. 2010, 6, 1968-1977.

6. Jin, R.; Moreira-Teixeira, L.S.; Dijstra, P.J.; van Blitterswilk, C.A.; Karperien, M.; Feijen, J. Chondrogenesis in injectable enzimatically cross-linked heparin/dextran hydrogels. J. Control. Release 2011, 152, 186-195.

7. Barbucci, R.; Giardino, R.; de Cagna, M.; Golini, L.; Pasqui, D. Inter-penetrating hydrogels (IPHs) as a new class of injectable polysaccharide hydrogels with thixotropic nature and interesting mechanical and biological properties. Soft Matter 2010, 6, 3524-3532.

8. Robert, L.; Robert, A.M.; Renard, G. Biological effects of hyaluronan in connective tissues, eye, skin, venous wall. Role in aging. Pathol. Biol. 2010, 82, 187-198.

9. Cowman, M.K.; Matsuoka S. Experimental approaches to hyaluronan structures Carbohydr. Res. 2005, 340, 791-809.

10. Smedsrod, B. Cellular events in the uptake and degradation of hyaluronan. Adv. Drug Deliv. Rev. 1991, 7, 265-278.

11. Ramli, N.A.; Wong, T.W. Sodium carboxymethylcellulose scaffolds and their physicochemical effects on partial thickness wound healing. Int. J. Pharm. 2011, 403, 73-82.

12. Okajima, K. Role of molecular characteristics on some physiological properties of cellulose derivatives. In Cellulose: Structural and Functional Aspects; Kennedy, J.F., Phillips, G.O., Williams, P.A., Eds.; Ellis Horwood: Chichester, UK, 1989; pp. 439-446.

13. Hirano, S.; Zhang, M.; Chung, B.S.; Kim, S.K. The N-acylation of chitosan fibre and the $\mathrm{N}$-deacetylation of chitin fibre and chitin cellulose fibre at a solid state. Carbohydr. Polym. 2000, $41,175-179$.

14. Hirano, S.; Nagamura, K.; Zhang, M.; Kim, S.K.; Chung, B.G.; Yoshikawa, M.; Midorikawa, T. Chitosan staple fibers and their chemical modification with some aldehydes. Carbohydr. Polym. 1999, 38, 293-298.

15. Kurita, K. Controlled functionalization of the polysaccharide chitin. Prog. Polym. Sci. 2001, 26, 1921-1971.

16. Park, J.H.; Saravanakumar, G.; Kwangmeuvung, K.; Kwon, I.C. Targeted delivery of low molecular drugs using chitosan and its derivatives. Adv. Drug Deliv. 2010, 62, 28-41.

17. Xu, T.; Xin, M.; Li, M.; Huang, H.; Zho, S. Synthesis, characteristic and antibacterial activity of N,N,N-trimethyl chitosan and its carboxymethyl derivatives. Carbohydr. Polym. 2010, 81, 931-936.

18. Liu, L.; Tang, X.; Wang, Y.; Gu, S. Smart gelation of chitosan solution in the presence of $\mathrm{NaHCO}_{3}$ for injectable drug delivery system. Int. J. Pharm. 2011, 414, 6-15. 
19. Sakai, S.; Hirose, K.; Moriyama, K. Control of cellular adhesiveness in an alginate-based hydrogel by varying peroxidase and $\mathrm{H}_{2} \mathrm{O}_{2}$ concentrations during gelation. Acta Biomater. 2010, 6 , 1446-1452.

20. Barbucci, R.; Pasqui, D.; Favaloro, R.; Panariello, G. A thixotropic hydrogel from chemically cross-linked guar gum: Synthesis, characterization and rheological behaviour. Carbohydr. Res. 2008, 343, 3058-3065.

21. Barbucci, R.; Leone, G.; Lamponi, S. Thixotrophy property of hydrogels to evaluate the cell growing on the inside of the material bulk (Amber Effect). J Biomed. Mater. Res. B Appl. Biomater. 2006, 76, 33-40.

22. Leone, G.; Fini, M.; Torricelli, P.; Giardino, R.; Barbucci, R. An amidate carboxymethylcellulose hydrogel for cartilage regeneration. J. Mat. Sci. Mat. Med. 2008, 19, 2873-2880.

23. Barbucci, R.; Fini, M.; Giavaresi, G.; Torricelli, P.; Giardino, R.; Lamponi, S.; Leone, G. Hyaluronic acid hydrogel added with hybuprofen lysine for the local treatment of condral lesions in the knee: In vitro and in vivo investigations. J. Biomed. Mater. Res. B Appl. Biomater. 2005, $75 B, 42-48$.

24. Omidian, H.; Park, K. Introduction to Hydrogels. In Biomedical Applications of Hydrogels Handbook; Ottenbrite, R.M., Ed.; Springer: London, UK, 2010; pp. 1-16.

25. Sederel, L.C.; van der Does, L.; Beugeling, T.; Feijen, J.; Bantjes, A.; Kim, S.W. Comparison of two carboxylate-activating agents for the modification of a synthetic heparinoid polyelectrolyte. J. Polym. Sci. Lett. Ed. 2003, 21, 1-11.

26. Barbucci, R.; Pasqui, D.; Giani, G.; de Cagna, M.; Fini, M.; Giardino, R.; Atrei, A. A novel strategy for engineering hydrogels with ferromagnetic nanoparticles as crosslinkers of the polymer chains. Potential applications as a targeted drug delivery system. Soft Matter 2011, 7, $5558-5565$.

27. Leone, G.; Delfini, M.; di Cocco, M.E.; Borioni, A.; Barbucci, R. The applicability of an amidated polysaccharidehydrogel as a cartilage substitute: Structural andrheological characterization Carbohydr. Res. 2008, 343, 317-327.

28. Park, J.W.; Choi, K.H. Acid-base equilibria and related properties of chitosan. Bull. Korean Soc. 1983, 4, 68-72.

29. Barbucci, R.; Magnani, A.; Lamponi, S.; Mitola, S.; Ziche, M. Cu(II) and Zn(II) complexes with hyaluronic acid and its sulphated derivative: Effect on the motility of vascular endothelial cells. J. Inorg. Biochem. 2000, 81, 229-237.

30. Mhetar, V.R.; Archer, L.A. A new proposal for polymer dynamics in steady shearing flows. J. Polym. Sci. Part B Polym. Phys. 2000, 38, 222-233.

31. Watanabe, H. Viscoelasticity and dynamics of entangled polymers. Prog. Polym. Sci. 1999, 24, 1253-1403.

32. Cuba-Chiem, L.T.; Huynh, L.; Ralston, J.; Beattle, D.A. In situ particle film ATR/FTIR spectroscopy of carboxymethyl cellulose adsorption on talc: Binding mechanism, $\mathrm{pH}$ effects and adsorption kinetics. Langmuir 2008, 24, 8036-8044.

33. Li, W.; Sun, B.; Wu, P. Study on hydrogen bonds of carboxymethylcellulose sodium film with two dimensional correlation infrared spectroscopy. Carbohydr. Polym. 2009, 78, 454-461. 
34. Haxaire, K.; Marechal, Y.; Milas, M.; Rinaudo, M. Hydration of polysaccharide hyaluronan observed by IR spectrometry. I. Preliminary results and band assignment. Biopolymers 2003, 72, 10-20.

35. Haxaire, K.; Milas, M.; Rinaudo, M. Hydration of polysaccharide hyaluronan observed by IR spectrometry. III. Structure and mechanism of hydration. Biopolymers 2003, 72, 162-173.

36. Trevors, J.T.; Pollack, G. Hypothesis: The origin of life in a hydrogel environment. Prog. Biophys. Mol. Biol. 2005, 89, 1-8.

37. Kasaai, M.R. A review of several reported procedures to determine the degree of N-acetylation for chitin and chitosan using infrared spectroscopy. Carbohydr. Polym. 2008, 71, 497-508.

38. Brugnerotto, J.; Lizardi, J.; Goycoolea, F.M.; Arguelles-Monal, W.; Desbrières, J.; Rinaudo, M. An infrared investigation in relation with chitin and chitosan characterization. Polymer 2001, 42, 3569-3580.

39. Nakanaga, T.; Sugawara, K.; Kawamata, K.; Ito, F. Infrared depletive spectroscopy of aniline-NH3 and aniline- $\mathrm{NH}_{3}{ }^{+}$clusters in a supersonic jet. Chem. Phys. Lett. 1997, 267, 491-495.

(C) 2012 by the authors; licensee MDPI, Basel, Switzerland. This article is an open access article distributed under the terms and conditions of the Creative Commons Attribution license (http://creativecommons.org/licenses/by/3.0/). 